\title{
Femoral artery pseudoaneurysm, an avoidable complication of femoral catheterization for hemodialysis
}

\author{
Mahesh Raj Sigdel
}

Department of Nephrology, Tribhuvan University Teaching Hospital, Kathmandu, Nepal

\section{Accepted on}

June $19^{\text {th }}, 2013$

\section{DOI Name}

http://dx.doi.org/10.3126/jaim.v2i2.8779

\section{Keywords}

femoral catheterization, hemodialysis, infection, pseudoaneurysm, surgical repair

\section{Citation}

Mahesh Raj Sigdel. Femoral artery pseudoaneurysm, an avoidable complication of femoral catheterization for hemodialysis. Journal of Advances in Internal Medicine 2013;02(02):65-6.

\footnotetext{
* Corresponding author

Mahesh Raj Sigdel, MD, Department of Nephrology

Tribhuvan University Teaching Hospital

Kathmandu, Nepal

Email: maheshsigdel@hotmail.com
}

\section{CASE}

The Nephrology unit got a call from Vascular Surgery department for evaluation and hemodialysis of a post operative patient. This was a 25 years lady on maintenance hemodialysis through radiocephalic arteriovenous fistula, prior to which she was hemodialysed through femoral access with multiple femoral punctures. She had complaint of painful and progressive right femoral swelling. At presentation, she was febrile with septic look, but systemic examination did not reveal source of infection. Local examination of right groin revealed a pulsatile, tender lump, $31 \times 27 \mathrm{~mm}$ in size. Immediate surgical exploration and repair of the same was done. Patient became increasingly septic and uremic. She was managed with intensive hemodialysis and antibiotics. After a stay of $>$ 3 weeks, she improved. She later on received renal allograft from her father and is doing fine till now. The attached image is of the groin wound. What was the underlying diagnosis?

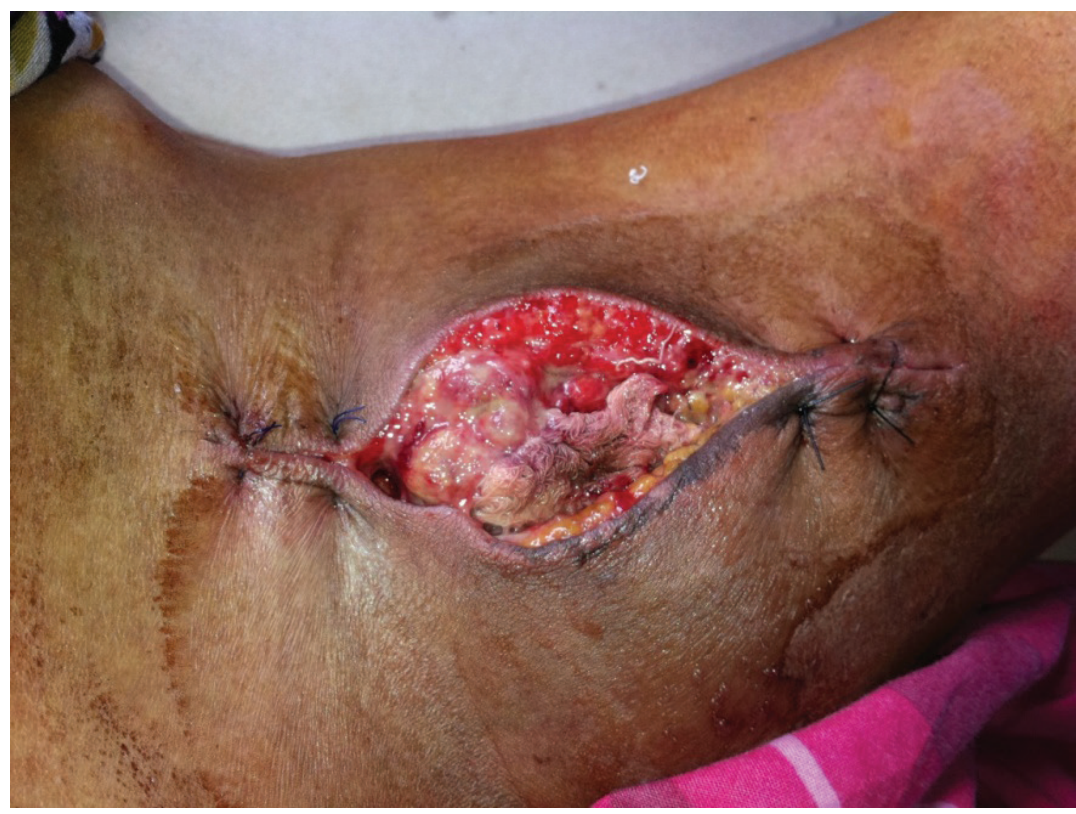

Figure 1. Surgical wound in the right groin

Answer: Femoral artery pseudoaneurysm following inadvertent arterial puncture during femoral venous catheterization.

Despite the fact that internal jugular access is preferred as temporary 
hemodialysis access, femoral catheters are still commonly used for hemodialysis, especially in developing countries including Nepal. In an unpublished observation from our hospital, $>90 \%$ of non elective hemodialysis is started through femoral access, reasons are manifold - clinical, technical and logistic. Though a relatively easy and benign procedure, femoral catheterization is not always safe. Complications range from arterial puncture, hematoma formation, infection and venous thrombosis to pseudoaneurysm formation, bowel penetration, bladder puncture, psoas abscess, septic arthritis, femoral arteriovenous fistula, femoral nerve injury etc. ${ }^{1}$

An arterial pseudoaneurysm is a pulsatile hematoma resulting from traumatic disruption of a portion of the arterial wall with leakage of blood into the surrounding tissue that is contained by fibromuscular tissue and communicates with the artery via a narrow neck. Use of anticoagulants, poorly controlled hypertension, hemodialysis, obesity, vasculopathy, improper techniques and inadequate compression are predisposing factors for pseudoaneurysm. It presents as painful pulsatile mass, usually beginning few hours after the procedure. Diagnosis is made based on history, finding of pulsation and bruit over the mass and doppler finding of "to-and-fro" flow. ${ }^{2}$ Complications include rupture (when $>3 \mathrm{~cm}$ ), infection, embolization, local pain, neuropathy and distal ischemia. Earliest management consists of prolonged \& strict bed rest, avoiding anticoagulation and manual compression. Subsequent options include ultrasound probe compression of the neck of pseudoaneurysm, intra-aneurysmal injection of thrombin and surgical repair; indications for surgical repair are rapid expansion of the pseudoaneurysm with risk of rupture, failure of percutaneous intervention, concomitant distal ischemia, neurological deficits and infected lesions. ${ }^{2}$

The steps to minimize complications of vascular procedure include appropriate training of the performers, use of sterile techniques, patient education and use of Doppler ultrasound to guide catheter insertion. ${ }^{3}$ It is advisable for nephrologists from developing countries to move away from femoral puncture, acquire the skill of jugular cannulation under ultrasound and fluoroscopic (C-arm) guidance and totally avoid repeated femoral route as a semi permanent dialysis access so that the dreaded complications like pseudoaneurysms discussed are largely mitigated.

\section{REFERENCES}

1. Kusminsky RE. Complications of central venous catheterization. J Am Coll Surg 2007;204:681-96.

2. Ahmad F, Turner S, Torrie $\mathrm{P}$, et al. latrogenic femoral artery pseudoaneurysms - a review of current methods of diagnosis and treatment. Clinl Radiol 2008;63:1310-16.

3. Gann M Jr, Sardi A. Improved results using US guidance for central venous access. Am Surg 2003;69:1104-7. 